\title{
Saber y hacer: evaluación de la transferencia de conocimientos didácticos a la práctica docente $^{1}$
}

\author{
Moira Alquézar² \\ Universidad Nacional de Mar del Plata, Argentina ${ }^{3}$ \\ moiralquezar@hotmail.com \\ Mariana Buzeki ${ }^{4}$ \\ Universidad Nacional de Mar del Plata, Argentina ${ }^{5}$ \\ marianabuzeki@hotmail.com
}

1 Artículo de reflexión.

2 Licenciada en Psicología, especialista en docencia universitaria.

3 Ayudante de Trabajos Prácticos en la Cátedra Adolescencia, Educación y Cultura. Docente de la Facultad de Humanidades, Universidad Nacional de Mar del Plata.

4 Licenciada en Psicología, especialista en docencia universitaria, doctora en trabajo social.

5 Jefa de Trabajos Prácticos en la Cátedra Adolescencia, Educación y Cultura. Docente de la Facultad de Humanidades, Universidad Nacional de Mar del Plata. 


\title{
Saber y hacer: evaluación de la transferencia de conocimientos didácticos a la práctica docente
}

\section{Resumen}

El presente trabajo pretende contribuir a la formación pedagógica de nuestros estudiantes mediante el análisis de uno de los dispositivos de evaluación propuestos por el equipo docente. El objetivo general es analizar el desarrollo de las clases organizadas por los estudiantes y su articulación con la propuesta didáctica de la asignatura «Adolescencia, Educación y Cultura». La metodología utilizada es de tipo cualitativo, enmarcada dentro de la investigación narrativa. El diseño es de corte naturalista: los datos se recolectaron en las clases prácticas, con la participación de todos los implicados en el campo. Las narraciones se recolectaron a través de un informe escrito de autoevaluación que los estudiantes debían presentar con posterioridad a la clase. Esta información se trianguló con las devoluciones que el grupo de la clase brindó a sus compañeros y con las observaciones realizadas por las ayudantes.

Palabras clave: Evaluación, prácticas en el aula, proceso de enseñanza-aprendizaje.

\section{Knowing and Doing: Transfer of Didactic Knowledge to the Teaching Practice Assessment}

\begin{abstract}
This research paper intends to contribute to the pedagogical training of our students by means of analyzing an assessment proposal made by teachers of the center. Our objective is to analyze the development of a series of sessions proposed by students; such sessions are articulated with what is seen in the subject: Adolescence, Education and Culture. A qualitative methodology is used in a narrative investigative frame. A naturalist design is also a key aspect: data collection was taken from practical sessions. Narrative collection was taken from selfassessment report due after class. This information was analyzed along with feedback provided by peers and observers.
\end{abstract}

Keywords: Assessment, Classroom practicum, teaching-learning process

\section{Saber e fazer: avaliação da transferência de conhecimentos didáticos à prática docente}

\section{Resumo}

O presente trabalho pretende contribuir à formação pedagógica de nossos estudantes mediante a análise de um dos dispositivos de avaliação propostos pela equipe docente. O objetivo geral é analisar o desenvolvimento das classes organizadas pelos estudantes e sua articulação com a proposta didática da matéria "Adolescência, Educação e Cultura". A metodologia utilizada é de tipo qualitativo, enquadrada dentro da investigação narrativa. O desenho é de corte naturalista: os dados coletaram-se nas classes práticas, com a participação de todos os implicados no campo. As narrações coletaram-se através de um relatório escrito de autoavaliação que os estudantes deviam apresentar anteriormente à classe. Esta informação se trianguló com as devoluções que o grupo da classe brindou a seus colegas e com as observações realizadas pelas ajudantes.

Palavras chave: Avaliação, práticas no sala, processo de ensino-aprendizagem. 


\section{Introducción}

La asignatura «Adolescencia, Educación y Cultura» pertenece al Ciclo de Formación Docente de la Facultad de Humanidades de la Universidad Nacional de Mar del Plata y se dicta para los profesorados de Documentación, Filosofía, Geografía, Historia, Letras y Ciencias Económicas. Se suele aconsejar a los ingresantes que la cursen al inicio de su carrera. Esto da lugar a que muchos de nuestros estudiantes sean aún adolescentes, situación que aprovechamos para que puedan identificarse y pivotear entre la posición del adulto —en su condición de futuros profesores - y la del adolescente — siendo ellos mismos, pero, a su vez, proyectándose como sus futuros alumnos- Asimismo, contamos con estudiantes que están cursando el último tramo de su carrera de grado, suceso posibilitado por el sistema de correlatividades vigente. Esta diversidad, en cuanto los estudiantes provienen de distintos profesorados, tienen distintas edades y se encuentran en distintos momentos de su formación, conforma, junto a las diferencias de historias, de estilos de aprendizaje, de capacidades, de dificultades, una heterogeneidad con la que nos topamos curso a curso. Aquí anclamos nuestra modalidad de trabajo en esa heterogeneidad, en esa diversidad, tomando como norte el desarrollo de la autonomía de los estudiantes y la formación y fortalecimiento de su pensamiento crítico. Evidenciados los cimientos, nos dedicaremos ahora a mostrar cómo pensamos y hacemos esta construcción.

Ofrecemos alternativas a los estudiantes para que puedan elegir entre diversas actividades en los procesos de enseñanza y de aprendizaje, como la posibilidad de optar por realizar un trabajo de campo o llevar adelante una clase. El trabajo de campo permite una aproximación a las problemáticas de los adolescentes desde el punto de vista de éstos. La clase, por su parte, brinda un primer acercamiento a la actividad del profesor, al manejo del aula y del rol docente. En este último caso proponemos la autoevaluación y la evaluación de pares, y en ambos procesos fomentamos las preguntas sobre el hacer y sobre el sentido de las tareas —-tutorías-; incentivamos la contextualización de los textos utilizados y de sus autores; consensuamos el encuadre de trabajo — estableciendo hojas de ruta al inicio del práctico—; enseñamos hábitos de estudio y trabajo — con las guías de lectura o síntesis de los materiales leídos-; proponemos la construcción de consignas de trabajo adecuadas —en la organización de la clase/trabajo de campo-; favorecemos el cuestionamiento del rol, de la tarea, de la función tanto propia como del otro — profesor/estudiante-. Así las cosas, priorizamos la adquisición de ciertas competencias que les permitirán continuar formándose a lo largo de su vida profesional.

El programa de la asignatura ha ido modificándose con base en la importancia que van cobrando ciertas problemáticas y teniendo en cuenta las sugerencias de los estudiantes en la encuesta anónima que deben entregar al finalizar de cada 
asignatura cursada; de ese modo, ha ido en aumento el espacio dedicado al tratamiento del problema de la violencia en sus distintas expresiones. También se han incorporado temáticas referidas a sexualidades y géneros para responder a las exigencias del sistema educativo en relación a la formación y capacitación de los futuros docentes para el abordaje de la sexualidad en la escuela. Con la aprobación de la Ley de matrimonio igualitario — Ley 26.618-, se sumó material relacionado a las identidades sexuales. Otro tema que cobra cada vez mayor relevancia es el de las nuevas formas de comunicación relacionadas al uso de nuevas tecnologías y las consecuencias que esto acarrea en los procesos de subjetivación. Además, hemos acrecentado el espacio dedicado al estudio de problemáticas psicosociales con las que el docente se ve enfrentado con frecuencia, tales como: adicciones, trastornos alimentarios, distintas formas de conductas de riesgo, etc. Es nuestra intención proveer a los alumnos de herramientas que les permitan dar respuesta a las demandas de los adolescentes y del sistema educativo. En concordancia con esta propuesta, el desarrollo de los trabajos prácticos en la cátedra intentó históricamente evitar que - a falta de lectura de los alumnos - se transformara en una clase expositiva en la que estaríamos pensando al alumno como un receptor pasivo de la información y en la que entraríamos en el círculo vicioso de «exponer porque los alumnos no leen y generar que los alumnos no lean porque el docente expone». Se hace necesario presentar el conocimiento disciplinar de modo que pueda ser elaborado y apropiado por aquellos a quienes está dirigida la práctica educativa.

Es nuestro propósito que los estudiantes construyan el conocimiento de la disciplina a través de la búsqueda personal de información y mediante procesos de resolución constructiva de problemas. Desde esta perspectiva, el docente debe estimular la actividad del alumno mediante la creación de circunstancias, de ambientes, que lo hagan posible. La comunicación con el alumno es una comunicación dialogada; se trata de una conversación estimulante, en la que la transmisión oral se combina con tareas de facilitación de fuentes, de orientación en su uso y en la resolución de problemas (Burbules, 1999). Tenemos en cuenta que, tal como se decía anteriormente, los alumnos que se inscriben en esta asignatura son, en general, ingresantes o se encuentran en el inicio de sus respectivas carreras. En la mayoría de los casos ésta es la primera asignatura del ciclo pedagógico que cursan. Esta situación hace que su primer contacto con el universo discursivo de la educación y la psicología educacional se haga a través de este espacio. Los estudiantes se hallan implicados en una doble acomodación: al régimen del discurso universitario - que se diferencia sustancialmente del modo discursivo de la escuela media- y a la formación discursiva del campo educativo/pedagógico.

Ha sido recurrente advertir que, aunque los estudiantes participan mucho, leen poco y, en su gran mayoría, presentan dificultades serias para escribir. Si bien esta cátedra ha diseñado instancias de evaluación que obligan a los estudiantes a organizar, sintetizar, analizar conceptos — tales como parcial domiciliario, trabajo de 
campo, organigrama de la clase a dar, trabajos prácticos—, y que los estudiantes han manifestado en las evaluaciones de la cursada que han aprendido mucho resolviendo estas actividades, somos conscientes de que las dificultades para comprender y para expresarse de forma escrita aparecen como evidentes en la instancia de evaluación. Entendemos que la entrada en la Universidad implica un giro importantísimo. De ahí que sea necesario «pensar de qué modo estamos implicados los docentes, las situaciones didácticas y las condiciones institucionales en las dificultades que encontramos en los alumnos»(Carlino, 2005, p. 45). Entendemos que leer y escribir implican una acomodación a modos discursivos particulares, que deben ser enseñados y no solamente exigidos en el momento de la evaluación. De este modo, procuramos ayudar a los estudiantes a ingresar en una comunidad tanto de discurso como de conocimiento especializado, y poner en acto la perspectiva constructivista del aprendizaje que se intenta transmitir en sus aspectos teóricos y en sus implicaciones prácticas y metodológicas.

\section{Fundamentación}

Reconocemos que la práctica docente es una actividad compleja en la que las preocupaciones habituales se centran en facilitar el aprendizaje, lo cual supone trabajar constantemente en la planificación de los procesos de enseñanza y de aprendizaje, en el enfoque metodológico, en el diseño de actividades, en instancias tutoriales y en la evaluación de los aprendizajes. Es en este último tema donde se inscribe nuestro trabajo. Entre los problemas que debemos enfrentar los docentes universitarios en los procesos de evaluación se destacan la falta de capacitación de los propios docentes en prácticas de evaluación no tradicionales, las falencias presentes en la formación previa de los estudiantes que ingresan a la Universidad, el desfase entre los métodos propuestos y las condiciones institucionales para llevarlos a cabo y el grado de incertidumbre que los jóvenes tienen con respecto a su futuro profesional. Hoy los cambios de empleo, de los perfiles profesionales, de los sistemas de acumulación y difusión de los conocimientos, hacen que estemos trabajando en una zona difusa. Díaz Barriga (2001) se refiere a la evaluación como el lugar de las inversiones. Desde su punto de vista, el examen es el instrumento que permite invertir los problemas sociales en pedagógicos, transformando un problema conceptual en un problema técnico. En la misma dirección, Litwin (2001) considera que la actitud evaluadora invierte el interés de conocer por el interés por aprobar, en cuanto se estudia para aprobar y no para aprender.

En la actualidad, los debates en relación a la evaluación de los aprendizajes giran en torno a entenderla, por un lado, como un proceso que debe orientarse al control externo de lo que hace el estudiante y a la mera calificación, y, por otro, como un proceso organizador e integrador de los aprendizajes. Si bien las concepciones sobre la evaluación han evolucionado paralelamente a la aparición de nuevos paradigmas y modelos educativos, el enfoque tradicional como medición de resultados y productos 
del aprendizaje predomina en función de homogeneizar y controlar los resultados obtenidos al final de los procesos de enseñanza y aprendizaje. En este sentido, el comúnmente denominado «examen» ha adquirido relevancia histórica como una forma de medir los conocimientos incorporados, y está habitualmente asociado a la idea de acreditación, es decir, la certificación de conocimientos curriculares previstos. Estudios en instituciones universitarias cubanas ponen en cuestión esta práctica evaluativa y reclaman su transformación (Gort, 2008; González, 2000). En la misma línea, investigaciones mexicanas de corte cuantitativo concluyen que la evaluación sigue efectuándose de forma tradicional, esto es, mediante la utilización casi exclusiva del examen, el cual consiste en la resolución de problemas, sin incluir algún instrumento de evaluación diferente. Además, los estudiantes declaran que siguen aprendiendo de manera memorística y no de forma significativa (Hernández et al., 2013). Investigadores brasileños señalan deficiencias y fragilidades en las prácticas de evaluación del aprendizaje, destacan el predominio de una evaluación básicamente sumativa y cuantitativa con escasa variedad de técnicas de evaluación utilizadas, y evidencian preocupación por la cantidad de contenidos que debe acumular el estudiante en sus aprendizajes, ausencia de planeamiento del proceso evaluativo y resistencia de los docentes para asumir cambios (Sampaio, 2012).

Frente a esta posición dominante emerge otra concepción que orienta la docencia con base en las estrategias centradas en el estudiante, y que requiere el desarrollo de competencias con el objetivo de dirigir la intervención pedagógica. Evaluar es mucho más que la evaluación de resultados del aprendizaje de los estudiantes, implica calidad de la enseñanza impartida.

Estudios referidos a la evaluación formativa, la evaluación mediadora, la evaluación auténtica, la evaluación grupal, la evaluación alternativa y por competencias, presentan puntos de convergencia y posibilitan la revisión o reflexión sobre nuestras propias prácticas. Investigaciones realizadas en Argentina (Celman, 2001; Litwin, 2001; Camilloni, 2001) destacan la importancia de implicar a los estudiantes en el proceso de evaluar su propio trabajo, por consiguiente, proponen que la evaluación debe requerir en algún momento una demostración activa de su capacidad de poner en acción el conocimiento, en contraste con hablar o escribir sobre él. Priorizan que durante el acto evaluativo se den oportunidades de clarificación, diálogo y discusión conjunta que sean revisadas en el mismo momento o en la siguiente actividad (Celman et al., 2012). Según diversos autores, la evaluación orientada al aprendizaje hace uso de técnicas de autoevaluación y de evaluación por compañeros, ya que éstas permiten que se impliquen, los compromete.

La evaluación auténtica es aquella que propone situaciones del mundo real o situaciones cercanas, problemas significativos, complejos, para que los estudiantes utilicen sus saberes previos, pongan en juego estrategias y demuestren la comprensión de los conocimientos nuevos. Todo este conjunto de evaluaciones puede englobarse 
bajo la denominación de evaluaciones alternativas, contraponiéndose de esta manera a las modalidades de evaluación tradicional. Las evaluaciones alternativas tienen como propósito que la evaluación se convierta en una autoevaluación tanto para el docente como para el estudiante. Estos enfoques intentan responder a la crítica que reciben los métodos de evaluación tradicional debido a su énfasis puesto en los resultados. Bajo esta orientación, Litwin (2001) caracteriza las buenas prácticas de evaluación como aquellas que satisfacen las siguientes condiciones: sin sorpresas, atractivas, enmarcadas en la enseñanza, sin desprenderse del clima, ritmo y tipo de actividades usuales de la clase; una perspectiva en la que la evaluación recupera la tranquilidad y proporciona seguridad a los estudiantes. Docentes y estudiantes difieren en los criterios con los que, entienden, se debe juzgar las evaluaciones, por ende, destaca el compromiso de transformar la evaluación en un acto de aprendizaje cuando los estudiantes comprenden y comparten los criterios con los que se realizó; al someterla al juicio de los estudiantes, se transparentan las aspiraciones o expectativas del trabajo docente.

En nuestra cátedra, desde hace tiempo, venimos implementando instancias de evaluación alternativas al examen tradicional, buscando que resulten acordes a lo que se pretende evaluar. De esta forma, si el objetivo es que los estudiantes aprendan a resolver situaciones problemáticas que se presenten en el desempeño cotidiano de su tarea docente, para saber si han logrado desarrollar esta capacidad, es necesario plantearles problemas reales e interesantes y no preguntas que midan simplemente su habilidad para retener los contenidos o establecer relaciones entre conceptos teóricos. Del mismo modo, si el objetivo es que incorporen una concepción constructivista de los procesos de enseñanza y de aprendizaje, la evaluación debe centrarse en la puesta en práctica de dicha concepción. Como señalan Piaget y Vigotsky, el aprendizaje comprende un proceso activo en el cual los estudiantes construyen significados a partir de experiencias previas. Entender la evaluación como instancia de aprendizaje implica organizar los dispositivos de tal manera que permitan a los estudiantes revisar y mejorar de forma progresiva sus producciones.

Los parciales domiciliarios y los comentarios de películas siguen una metodología secuencial que se dirige en ese sentido. Primero, las consignas se trabajan en clase en pequeños grupos, con puesta en común y devolución de las docentes; luego se realiza la actividad domiciliaria, que es corregida y devuelta a los estudiantes con observaciones; finalmente, deben reescribir el trabajo, autoevaluarse y justificar la calificación que se asignaron.

Otras actividades alternativas de aprendizaje y evaluación son el trabajo de campo y la clase. Ambas se realizan en grupos pequeños. En esta ocasión nos remitimos a la segunda, la cual se lleva a cabo en los prácticos. Los estudiantes a cargo deben estructurarla desde una concepción constructivista, proponiendo actividades y tareas que promuevan la participación activa en la construcción del conocimiento. 
Los temas a tratar en cada clase, la bibliografía correspondiente y la fecha en que tendrá lugar figuran en el cronograma que reciben al comienzo de la cursada. La distribución de las clases se organiza a elección de los propios alumnos, que pueden, si lo desean, agregar bibliografía ampliatoria. Cuentan con tutorías previas para el armado y organización de las actividades. Los últimos quince minutos de cada clase se reservan para la evaluación y autoevaluación de la misma: primero, de quienes estuvieron a cargo de la clase, posteriormente de los compañeros y, por último, de las ayudantes, quienes señalarán debilidades y fortalezas, errores y aciertos.

Partiendo de nuestra experiencia como ayudantes de trabajos prácticos hemos observado que los estudiantes presentan dificultades en la aplicación de conocimientos teóricos, asimilados durante la cursada, a la resolución de problemas prácticos, incluyendo las clases, las cuales a menudo siguen siendo expositivas. Algunas modificaciones orientadas en este sentido nos permiten afirmar que el trabajo colaborativo, ya sea en pequeños grupos o en discusiones grupales, ha mejorado significativamente sus producciones. Esto nos ratifica que la interacción con otros en todas las instancias, abarcando las de evaluación, constituye un cimiento de gran relevancia en los procesos de enseñanza y aprendizaje.

Notamos actualmente un doble discurso: por una parte, se establece la necesidad de atender la diversidad de los estudiantes en el aula y, por otro, se manejan propuestas únicas para poblaciones homogéneas inexistentes. Este es el conflicto que enfrentamos y que intentamos problematizar desde nuestra propuesta pedagógica, proponiendo distintos caminos para llegar a una misma meta, y queda traducido en el siguiente objetivo general: analizar el desarrollo de las clases organizadas por los estudiantes y su articulación con la propuesta didáctica de la asignatura; y en los siguientes dos objetivos específicos: identificar el modelo didáctico puesto en práctica en el empleo de recursos, contenidos y actividades por los estudiantes que organizan la clase, e identificar qué conocimientos abordados en la cursada se han transferido a la práctica docente.

\section{Metodología}

La metodología que se utilizó en este estudio es de tipo cualitativo y se enmarca dentro de la investigación narrativa, pues la interpretación y la comprensión adquieren relevancia y se centra la atención en los aspectos subjetivos (Valles, 2000). Nuestras prácticas se fundamentan en un enfoque cualitativo que implica la intencionalidad de conocer y comprender críticamente los objetos en los procesos evaluativos, entendiendo que son los sujetos quienes los construyen y reconstruyen (Celman et al., 2012) La perspectiva narrativa permite dar sentido a las propias acciones y construir los propios significados, lo que hace inteligible para el narrador aquello que relata (Clandinin 2006; McEwan, 2005). Bruner (1991) afirma que la narración es una modalidad de pensamiento que permite organizar la experiencia. 
Al narrar adoptamos una perspectiva, contamos los acontecimientos a partir de una determinada posición y vamos construyendo una trama que supone una mirada que enuncia ciertos aspectos. Es decir, una misma serie de acontecimientos puede dar lugar a distintos relatos construidos por la misma persona en diversos momentos, esto genera una nueva configuración que posibilita observar otros significados.

La perspectiva narrativa deja reconstruir y reorganizar la experiencia a través de los relatos, atribuyendo significados al pasado, con referencia al presente y a las concepciones personales. Las circunstancias acontecen en los contextos sociales, culturales e institucionales que les otorgan sentido y se interpretan de acuerdo a la interacción de los sujetos con su entorno (Álvarez y Porta, 2012, p. 81). El pensamiento narrativo permite no sólo un análisis crítico, sino que también propicia el diálogo entre diversas perspectivas, competencias y formas de pensamiento. Es una herramienta para pensar acerca de la experiencia, penetrar en la identidad, los significados y el saber práctico (Álvarez, Porta y Sarasa, 2010). Esta perspectiva resulta especialmente útil en la investigación educativa, porque la narración aborda lo que se hace, lo que el narrador interpreta acerca de su acción y lo que interpretan aquellos con quienes interactúa, en este caso, las investigadoras (Erickson, 1997).

El diseño es de corte naturalista dado que la investigación se realiza en el mismo contexto que se pretende estudiar y no en condiciones experimentales: la información se recolectó en las clases prácticas, con la participación de todos los implicados en el campo. Las fuentes de datos fueron los informes de autoevaluación -38- de quienes organizaron la clase, los cuales consistieron en un relato de su implicación —cómo ellos mismos experimentaron su rol docente y lo sucedido en el transcurso de la actividad-. Estos informes se triangularon con las observaciones de los compañeros que participaron como "grupo-clase» y las de las ayudantes. La muestra se conformó por treinta y ocho estudiantes, de todos los profesorados de la Facultad de Humanidades, que cursaron la asignatura durante el segundo cuatrimestre de 2013 y que optaron por el dispositivo de evaluación «dar clase». Para la observación de las clases se utilizaron indicadores que fueron elaborados y consensuados entre el equipo docente y los estudiantes. Los criterios fueron los siguientes: distribución del tiempo en la clase, organización de la tarea, actividades seleccionadas, coordinación de los grupos de trabajo y coordinación entre los integrantes del grupo — aspectos formales-; y recursos utilizados, adecuación de las estrategias utilizadas, conocimientos del tema, vinculación con conocimientos previos/otros — aspectos pedagógicos—. Para la preparación de las clases se ofreció un espacio de tutorías, no aprovechado por la mayoría. Ulteriormente realizamos análisis de contenido de las autoevaluaciones; nos dedicamos a la tarea de identificar criterios utilizados en los registros de autoevaluación, en la construcción de categorías y en el análisis de la información obtenida desde un enfoque interpretativo de los significados de los propios actores (Goodson, 2005; Clandinin, Steeves y Chung, 2008). Cuando se investiga desde el enfoque interpretativo, se ponen en juego dos 
narrativas: las que hacen los sujetos acerca de sus prácticas —los estudiantes que dan clase a sus pares-y las que hacemos los investigadores (Vain, 2012).

\section{Análisis de las clases}

Teniendo en cuenta que los procesos de enseñanza y aprendizaje implican casi siempre tensión entre teoría y práctica, mediante el diseño de esta actividad, intentamos acortar la distancia entre lo que se enseña y lo que se aprende. Pretendemos aliviar la ansiedad e incertidumbre que genera «la realidad de la práctica docente» por su carácter diverso, complejo y particular, y acompañar esta vivencia con crítica reflexiva, fundamentación y relaciones significativas de integración con conceptos brindados en el inicio de la cursada. Reconocemos que la evaluación presenta complejidades, momentos conflictivos y negociaciones; sin embargo, pretendemos, o al menos intentamos, que la evaluación de la actividad sea parte de la enseñanza y del aprendizaje e implique para los estudiantes una mirada reflexiva acerca de sus propios aprendizajes. Explicitar y socializar los procesos que se pusieron en juego, dudas, pensamientos e ideas que condujeron a la realización de la clase o cómo fueron ordenando el tema conducen a la formación de sujetos creativos, autónomos y críticos en relación al conocimiento que enseñan y aprenden. La reflexión y la problematización de la propia práctica, la propuesta inicial, actividades realizadas, logros alcanzados — previstos o no-, facilita la tarea de establecer relaciones y argumentar decisiones. Se trata de tomar conciencia sobre los procesos que se ponen en juego al aprender y reflexionar acerca de la pertinencia de las estrategias de aprendizaje y el manejo de los contenidos seleccionados, así como de la eficacia del tiempo y el esfuerzo empleado; se trata, en fin, de ayudar a pensar por qué hacemos lo que hacemos contribuye a solucionar inconvenientes de la práctica en su devenir y aliviar la ansiedad del ejercicio como profesor. Abrir el diálogo favorece el descubrir que pueden pensar y aportar a la construcción de la propia práctica y al rol docente. La experiencia de la evaluación conjunta y la discusión con los estudiantes de los criterios de evaluación nos permite repensar que ésta es una experiencia en la que los estudiantes aprenden in situ. Al decir de Celman (1993):

[...] el aprendizaje [se entiende] como un proceso reflexivo, en el cual no sólo se aprende contenido sino maneras de aprender y pensar, mediante procesos interactivos individuales y grupales. $\mathrm{Y}$ el docente, como profesional reflexivo, creativo, con capacidad de «recuperar el pensar del hacer» (p. 13).

El hecho de no haber separado los comentarios de los estudiantes que dieron las clases, de sus compañeros y de nuestra observación, es intencional porque la evaluación de la transferencia del conocimiento teórico a la práctica docente se construyó de forma colectiva e incluyó los aspectos privilegiados por todos los implicados. Algunos aspectos observados durante las clases desarrolladas por los estudiantes, señalados por sus pares, son: ilustración de los contenidos teóricos por medio de videos, documentos, artículos periodísticos, entrevistas, etc.; dificultades en la organización del tiempo, en la 
distribución de las tareas, en la coordinación del debate y en el manejo de la palabra e inconvenientes para reconducir la clase hacia la apertura de problemáticas vinculadas con la temática principal. A estas dificultades, que nosotras hemos corroborado, le sumamos otra que es la imposibilidad de los estudiantes de efectuar una evaluación exhaustiva y profunda acerca del rol docente que desempeñaron los compañeros.

A partir de las observaciones efectuadas por las docentes $-\mathrm{y}$ por los propios compañeros - y de la lectura de los autoinformes individuales se elaboraron las categorías que presentamos a continuación:

- Tiempo: refiere al manejo y organización que el grupo hace del tiempo en relación al inicio, desarrollo y cierre.

- Equipo docente: abarca la distribución de los roles y de las tareas al interior de cada grupo y la coordinación con respecto al grupo-clase.

- Recursos utilizados: soporte material utilizado durante la clase.

- Estrategias didácticas: adecuación y pertinencia de las actividades diseñadas para lograr los objetivos previstos.

- Conocimiento del tema elegido: refiere a la vinculación con conocimientos previos de la asignatura tanto como con otros conocimientos disciplinares.

Uno de los aspectos abordados es el tiempo. Si bien destacamos la importancia de organizar el devenir de la clase y entendemos el peligro de la improvisación absoluta, señalamos que tanto la imprevisibilidad como la incertidumbre son parte inevitable de este proceso, sabiendo que cada propuesta grupal sufre las modificaciones que surgen de las inquietudes de los estudiantes, suscitadas en el momento. No obstante, el diseño de los objetivos y las actividades planificadas intentan contemplar diferentes niveles de realización. La mayoría de los estudiantes le otorgaron un papel central a esta categoría, tanto para el diseño como para la puesta en práctica de la clase. Pero la dificultad se presentó en el ajuste, es decir, en la adecuación de las actividades «en» el tiempo. En este sentido, nosotras observamos diversos inconvenientes: algunos estudiantes se manejaron extensamente en el inicio de la clase, no pudiendo dedicar tiempo a la puesta en marcha de la actividad; algunos, hicieron súbitamente una entrada a la actividad con dificultades para efectuar un cierre; otros, quedaron superados por la gran cantidad de recursos implementados y desbordados con el tiempo; y por último, otros tantos resultaron desfasados en el tiempo debido a las dificultades con el soporte tecnológico, que generó una alteración difícil de superar: 
El tiempo nos jugó en contra (Ai 2). ${ }^{6}$

No logramos adecuar las actividades propuestas con el tiempo (Ai 3).

Estaba con la hora bastante presente para que no se hiciera muy extensa la exposición del tema (Ai 9).

Llegamos un poco justas con el tiempo (Ai 14).

El fuerte de la clase estuvo en la organización y manejos de los tiempos en los que fueron distribuidas las tareas (Ai 16).

Tuvimos problemas técnicos pero logramos seguir (Ai 34).

Con la implementación de este dispositivo les sugerimos a los estudiantes la modalidad de trabajo grupal, pues consideramos que promueve el aprendizaje profundo y además posibilita confrontar las propias ideas con las ajenas al interior del mismo. Sin embargo, luego de la selección de los temas, algunos estudiantes quedaron agrupados en pequeños grupos y otros pocos Ilevaron a cabo la actividad de forma individual. En estos últimos casos, nosotras observamos, en coincidencia con los comentarios de los estudiantes, que estas clases giran alrededor del profesor, quien recapitula, pregunta, ejemplifica y explicita pensamientos, manifiesta su posición y transparenta sus formas de pensar el diseño de la clase, buscando la interacción y participación con los alumnos. Empero, resulta difícil apartarse de ese nivel expositivo para construir en conjunto el conocimiento, construcción que se queda en un plano tentativo, es decir, de aproximación: «dar más espacio a la discusión, dando el tiempo para que mis compañeros pudieran dar sus puntos de vista en torno al tema» ( $\mathrm{Ai} 32$ ); "no fue una clase expositiva, pero tampoco constructivista» (Ai 8).

En relación a las clases grupales, vemos cómo el trabajo colaborativo brinda sus fortalezas y debilidades. Entendemos que un trabajo colectivo supone intercambios, selección de ideas y acuerdos, solidaridad, para el logro de una producción común; aquí no sólo es importante el producto final sino el proceso que el grupo va realizando en las distintas etapas, como establecer objetivos, tiempos, selección de recursos, distribución de roles y funciones, etc. Pero también es cierto que algunos estudiantes encuentran la oportunidad de refugiarse en el grupo para ocultar su inactividad, generando en las ayudantes ciertas inquietudes al momento de efectuar la evaluación y la calificación.

De otro lado, observamos que la tendencia es que la conformación de los grupos se realiza por criterios de afinidad: los miembros se conocen mutuamente, provienen, en general, de la misma carrera, ya tienen hábitos comunes de estudio y

6 La expresión «Ai» significa «autoinforme»; así, se tiene que «Ai 2» es «autoinforme dos»; «Ai 3», «autoinforme tres»y así sucesivamente. 
de trabajo, lo que facilita la organización del trabajo grupal en todos sus aspectos. Un grupo minoritario queda unido al azar por diversos motivos. Inferimos que a mayor cohesión grupal mejor desenvolvimiento y producción colectiva que se transfiere al manejo del grupo-clase; en clave de Watzlawick (1985): «la totalidad es más que la suma de las partes».

Queda abierta la indagación respecto al porqué ese plus no se genera en los grupos rearmados de acuerdo a otros criterios — como, por ejemplo, que alguien haya quedado sin compañero-, en los que existe el interés pero no resulta suficiente para generar una clase de otra índole. En esta construcción intersubjetiva se apunta a conocer algo que nos implica con el otro, y a los otros como sujetos del campo, en una situación que se reconoce compleja, así como se busca el encuentro con el otro de sí mismo; ver-se en el otro es lo que permite constituir la práctica en «experiencia de sí», interiorización y transformación de uno mismo a través de una práctica.

Yo no hablé mucho y mi compañera tenía una actitud más participativa (Ai 15).

Al diseño de la clase le faltó coordinación y fue librado a la espontaneidad del debate (Ai 29).

No pudimos relacionar el tema a tratar con los autores y no pudimos ocultar la falta de un compañero, porque no pudimos cubrir la parte que a él le tocaba (Ai 31).

Para diagramar la clase no tuvimos inconvenientes, nos fue fácil trabajar y entendernos (Ai 13).

En coincidencia con las narrativas de los estudiantes, nosotras hemos observado que en estas clases los futuros docentes han logrado plasmar en distintos grados algunas ideas constructivistas; no obstante, fue difícil destituir el eje tradicionalmente organizador de la exposición. Nos surgen algunas reflexiones al respecto. Por un lado, aparece la idea instalada del docente como proveedor de conocimiento, que le permite mantener el control y la seguridad acerca de los cuestionamientos y planteamientos de los estudiantes. Asimismo, reconocemos que ésta es una primera aproximación al rol de la tarea docente, por tanto, la inexperiencia se hace presente. Somos conscientes de la tensión a la que los invitamos a participar: cuestionar el quehacer docente en un "como si» y la puesta en marcha de la clase diseñada da como resultado una distribución de roles y funciones al estilo de una conocida exposición oral, lejos del modelo constructivista que pretendemos que interioricen.

[Se] nos dificultaba no hacer una clase expositiva (Ai 6).

La clase estuvo organizada para que no sea expositiva, pero no se armó una clase del tipo constructivista (Ai 7).

No es lo mismo dar una exposición oral a planificar y llevar adelante una clase ( $\mathrm{Ai}$ 18). 
Otro de los elementos analizados consistió en los recursos seleccionados por los estudiantes y la utilización de los mismos. Hallamos una cierta inclinación a suponer que lo innovador en una clase áulica pasa por los recursos tecnológicos utilizados y no por el sentido, la finalidad, la toma de posición con que éstos se utilizan. Nuestros estudiantes se caracterizan por ser lo que se ha denominado «nativos digitales de esta era virtual», y si bien esta diversidad de soportes puede resultar propicia para un aprendizaje - ya que potencian nuevas formas de producir conocimientos, leer e interpretar la realidad-, es necesario resaltar los aspectos cuestionados a los que el uso no reflexivo conlleva, que interpelan el «para qué» del soporte. En las clases observadas se puso en evidencia lo que Inés Dussel (2011) plantea como cierto empobrecimiento o superficialidad de las estrategias cognitivas empleadas con la introducción de los nuevos medios digitales en las aulas. La gran mayoría de las clases fueron diseñadas con un repertorio variado de medios tales como tiza y pizarrón, bibliografía obligatoria, videos, fragmentos de películas, artículos periodísticos, letras de canciones, fotografías, collage, encuesta anónima, guía de preguntas; y sin embargo, detectamos un limitado uso crítico y estratégico de los recursos en su doble dimensión: como apoyo a la enseñanza — con posibilidad de ser asociado a otras estrategias - y como contenido de la enseñanza en sí mismo (Anijovich y Mora, 2012), sin dejar de lado las dificultades que trae aparejadas la no previsión del funcionamiento y del soporte técnico necesario. Se suelen adoptar secuencias didácticas similares que consisten en la explicación, el uso del pizarrón y, después, la utilización del recurso tecnológico como forma de ampliar lo que se dijo oralmente o ilustrar los contenidos de los textos. Igualmente, también es cierto que existe una creencia que establece una relación directa entre recursos tecnológicos, diversión y motivación, la cual admite que el entusiasmo del estudiante favorece la predisposición para el trabajo en conjunto y, consecuentemente, el aprendizaje: «Tratamos de buscar la manera de atraerlos» (Ai 10); «tuvimos problemas técnicos pero logramos seguir» (Ai 34); «el resultado de la encuesta iba a obrar como una suerte de fuerte disparador, pero pasó casi inadvertida»(Ai 27). Profundizando en esta dirección de análisis, la participación efectiva de los estudiantes tiene muchas aristas que van desde los materiales y recursos de apoyo, el tipo de contenidos que prioricen, actividades que realicen, el nivel de complejidad de las mismas, como las posibilidades de opción que generen.

Continuamos el análisis vinculando las siguientes categorías y estrategias didácticas con el soporte material y conocimiento del tema elegido e interacción con el grupo-clase. En relación a estos aspectos surgen situaciones bastante variadas y, por este motivo, decidimos centrarlas en cuatro escenarios vivenciados que ubicamos en una misma línea de trabajo, enunciando desde los aspectos más rudimentarios hasta las experiencias más enriquecedoras para la construcción conjunta del rol docente.

El primer escenario es el representado por los grupos en los que faltó comprensión de las ideas nodales de la bibliografía: las actividades propuestas resultaron estar 
desconectadas de los contenidos y ser pobres, lo que generó poca interacción, ausencia de debate. En las situaciones en las que no se diseñó actividad, la participación oscila entre escasa y nula, y la intervención del docente generó un clima de pseudocomunicación. Abundaron las ejemplificaciones aportadas por el equipo docente, no se evidenció un tratamiento profundo de los contenidos y se careció de conexiones con otros conocimientos. El efecto fue el de diálogos poco profundos, poco debate y falta de promoción del juicio crítico. La ausencia de comprensión cabal del concepto central que estructura cada texto desvirtúa el abordaje del tema y se sostiene, en cambio, un intercambio superficial, de sentido común, más referido a la vivencia personal que al arribo de una construcción grupal de mayor abstracción. Los «docentes» no lograron ni coordinar ni guiar el diálogo intenso, y la participación apasionada que se despertó en algunas ocasiones tampoco pudo, en general, reorganizar la abundante información que fueron aportando los estudiantes: " "Me siguen", "queda claro", "entienden"» (Ai 1); "comencé con preguntas para que se familiaricen con el tema y se cree un ambiente de confianza en el que participen todos» $(\mathrm{Ai} 4)$.

Algunos logran sostener el interés de los estudiantes, pero el resultado es el mismo; o tienen buena predisposición para recepcionar inquietudes, escuchan, pero no reconducen la clase a otro nivel de problematización: «En algunos casos sentí que las preguntas quizás no estuvieron bien redactadas o que no apuntaban directamente a una discusión» (Ai 19); "primero la duda era cómo comenzamos y más tarde se sumó: ¿cómo terminamos?» (Ai 25).

Otro escenario observado fue el de aquellos estudiantes que, en el rol docente, lograron una buena comprensión del texto y realizaron una adecuada selección de aspectos centrales y aleatorios —una selección acorde a la actividad-, buena disposición de trabajo con los pequeños grupos, aquellos que, en fin, captaron y mantuvieron el interés de los estudiantes y su participación. Sin embargo, la dificultad se presentó en la puesta en común —a la que no se arribó-, lo cual no posibilitó el debate ni la promoción del juicio crítico, ello debido a la abundancia de recursos implementados que no cumplieron la función con la que fueron seleccionados; esto imposibilitó trabajar profundamente, produciendo una ilustración del tema más que una construcción conjunta del conocimiento: «Para enseñar debíamos comprender» (Ai 3); «fallamos por seguir el debate por separado y no grupalmente» (Ai 3); "[los contenidos] iban quedando descolgados, a tal punto que no sabía cómo introducirlos a la clase» (Ai 38).

Un tercer escenario fue el presentado por los que tuvieron inconvenientes en el rol docente para identificar las diversas posiciones a las que se refería el tema central, realizando una selección parcial de la bibliografía, con apoyo en una sola postura, ofrecida con buena comprensión, pero sin promover el debate o el juicio crítico. Inclusive, no se dio lugar a una postura diferente del estudiantado, no se 
problematizó la tensión del tema, lo que impidió otra línea de discusión alternativa a la postura sostenida por el equipo docente. En la puesta en común de la producción grupal surgieron diversos puntos de vista u opiniones que los docentes no lograron clarificar, organizar ni regular. Debido a este proceso, les resultó difícil hacer un cierre contemplando la pluralidad de puntos de vista de la problemática planteada. Se lograron generar conocimientos nuevos, pero con las dificultades mencionadas.

No pudimos generar debate (Ai 5).

Opino que tuvimos tres problemas: los nervios, la tarea que propusimos, y la falta de debate. [...] podríamos haber redactado de una manera más clara las consignas... esto podría haber generado algún tipo de discusión más profunda (Ai 6).

No resolvimos el total encauzamiento del debate (Ai 14).

El debate por momentos se nos fue un poco de tema (Ai 28).

Un cuarto escenario corresponde a aquellos que lograron articulación y coherencia en la comprensión cabal de los contenidos, puesto que los jerarquizaron y los organizaron, así como ajustaron el soporte y la estrategia con actividades y consignas adecuadas, con participación de los estudiantes e intervenciones reguladoras del equipo docente que condujeron a la promoción de conocimientos nuevos y juicio crítico. Sólo se observaron algunas dificultades a la hora de ofrecer un cierre, la elaboración de algunas conclusiones y las cuestiones a seguir trabajando.

Se mantuvo un espíritu democrático y de respeto a las opiniones de los demás durante el desarrollo de la clase (Ai 16).

[Se trató] en todo momento de no exponer el tema, sino que el mismo surja de la generación de temas que el debate producía (Ai 17).

Otra cosa que me gusta mucho [de] como nos salió fue el generar el debate, el poder hacer que alumnos que en otras clases no hablaban pudieran participar (Ai 18).

Respecto a la interacción producida entre el equipo docente y el grupo-clase, la mayor tendencia fue el diseñar la clase con una propuesta de trabajo grupal, ya fuera que la resolución de la actividad la llevaran a cabo en pequeños grupos, en parejas o en el grupo mayor. Es preciso destacar que si bien se evidenció una intención pedagógica de talante constructivista —entendiendo que la intención cooperativa entre alumnos puede resultar potenciadora del aprendizaje-, ello sólo es posible bajo ciertas condiciones, pues poner a los alumnos a trabajar en grupos no garantiza per se la maximización de la actividad constructiva. Existieron desajustes al interior de los equipos docentes, visibles en las dificultades para adecuar tanto el tiempo como el desarrollo de la actividad, quedando manifiestas las falencias en esta interacción. Esto fue percibido por los mismos equipos, por el grupo-clase y por nosotras. Por ejemplo, notamos que hubo escaso monitoreo de las producciones 
grupales para contrastar si se desarrollaban en las direcciones sugeridas, una tendencia a acercarse sólo al grupo más demandante o al que más trabajaba, y no se ofreció al resto la oportunidad de aportar clarificaciones, despejar dudas y orientar la discusión: "Quizás tendría que haberle prestado la misma atención a todos» (Ai 10); «no tuvimos en cuenta el manejo de un grupo grande de alumnos» (Ai 9).

En un plano general, la vinculación con conocimientos previos se pretendió lograr a través de la implementación de varias estrategias, desde lluvias de ideas, definiciones intuitivas, preguntas de apertura, cuestionarios, mapas conceptuales, entre otras herramientas, pero consideramos necesario destacar que el inconveniente residió en la escasa conexión con el nuevo contenido, sin recapitulación profunda posterior. Situaciones similares se encontraron cuando se pretendía realizar articulaciones con conocimientos de otras disciplinas o conocimientos impartidos en nuestra asignatura. Pensamos que esta pesquisa les resultaba útil al momento de pensar la organización de la clase, pero no logró cumplir su finalidad: tomar conciencia de lo que el estudiante sabe —sus contenidos erróneos e ideas intuitivas sin organización-, no funcionó como un enganche del nuevo contenido. En síntesis, la debilidad se manifiesta en la no construcción conjunta, ni siquiera a partir del obstáculo, para someter al conocimiento erróneo a una deconstrucción, ni como puente que acerque lo familiar a lo novedoso.

Como observadoras, partimos del presupuesto de que las mayores dificultades surgirían en las primeras clases. Empero, también creíamos que las características del primer grupo generarían una buena base orientativa para el resto. No obstante, algunas clases posteriormente desarrolladas no lograron incorporar las sugerencias provenientes de las devoluciones de las clases anteriores, de los compañeros y de las docentes a cargo. Es interesante hacer mención de la preocupación en relación al recorte que necesariamente tuvieron que realizar al diseño original y sin advertir que ello los condujo a un arribo igualmente satisfactorio, lo cual dio cuenta de los niveles de exigencia que muchos traen. Asimismo, otra preocupación, esta vez generalizada, comprende dos aspectos: uno consistió en que no «pudieron exponer todo lo que sabían» y, el otro, las dificultades presentadas en el momento de efectuar el cierre de la clase. Creemos que una posibilidad de articular las preocupaciones a las que ellos hacen referencia «reside» en el momento de cierre, en el que se puede utilizar la exposición como forma de reconceptualización, recapitulación, activación de procesos cognitivos, replanteo y apertura de conocimientos, es decir, operando como síntesis del tema:

No fue muy igual al diagrama que habíamos organizado... para no perdernos (Ai 2).

Existen muchos puntos por mejorar y muchos otros por comprender, pero creo que eso tiene que ver con la formación docente (Ai 3).

Mi clase fue realizada fijándome en los errores de la clase anterior (Ai 5). 
Surgieron cosas imprevisibles, no sabes para qué lado va a salir el tema. [...] no sabes si vas a estar a la altura de la situación (Ai 7).

La devolución muy buena, me abrió un abanico de posibilidades para, en su momento, preparar otra clase (Ai 25).

Como error me sirvió para la próxima oportunidad que tenga de dar clase (Ai 26).

Simultáneamente a todos estos replanteos que emergieron, no podemos dejar de contemplar la riqueza a nivel afectivo que el dispositivo despierta en los estudiantes, tanto en su grado de cuestionamiento como de satisfacción, lo que consolidó esta experiencia como una primera aproximación a la tarea docente:

Fue una experiencia enriquecedora, me hizo dar cuenta que tengo mucho por aprender, mejorar y que realmente esta es mi profesión (Ai 4).

Al principio estaba muy nerviosa... después el grupo me dio la confianza necesaria (Ai 10).

Es algo que me hizo sentir muy bien y sin duda alguna quisiera repetir (Ai 15).

Confirmo con cada clase mi vocación docente (Ai 24).

Lo tomo como puntapié para mejorar la profesión que elegí, que es ser docente (Ai 32).

\section{Consideraciones finales}

Para todos nuestros estudiantes fue la primera experiencia en relación al rol docente. Confirmamos que las clases dadas en forma grupal logran mayores beneficios y consolidan los procesos de enseñanza y de aprendizaje. Ahondando en el propio dispositivo, observamos que la dimensión temporal juega un papel clave en este proceso, ya que interviene modelando o distorsionando los distintos momentos de la clase. Destacamos que, si bien se han logrado plasmar algunas ideas constructivistas, fue difícil destituir el eje tradicional organizador de la exposición e ilustración de conocimientos. Entendemos que esta dificultad en la práctica obedece a modelos internalizados, naturalizados y escasamente interpelados; pensamos en un continuum entre la clase expositiva, en un extremo, y la clase constructivista, en el otro, y en el que los distintos grupos oscilaron con diversos grados en este proceso. La riqueza de la vivencia radica en «jugar a ser docente» y en la instalación de un espacio de reflexión sobre la propia formación. Se comprende que la identidad del «ser» docente parte del reconocimiento de la reflexión sobre uno mismo como herramienta que posibilita generar cambios en el proceso de enseñanza y, por consiguiente, también en el aprendizaje. 
Otro punto a destacar es la dificultad para recortar los contenidos relevantes del tema a tratar, situación que conlleva a una insuficiente comprensión de la bibliografía, lo cual desdibuja las estrategias seleccionadas, las consignas elaboradas, y provoca una escasa articulación con el conocimiento del tema, lo que da como resultado discusiones superficiales, clases poco flexibles en su estructura y con inconvenientes para vincular con conocimientos previos, orientar el debate, clarificar posturas y promover el juicio crítico.

Por otra parte, queremos destacar algunas cuestiones en relación a los informes de autoevaluación que los estudiantes debían entregar la semana siguiente a la clase en la que participaban como docentes. En dichos informes se les solicitó que, luego de la autoevaluación individual, pudieran pensar una nota con la que se calificarían. Observamos que hubo una coherencia entre la percepción de la autoevaluación y la evaluación realizada por nosotras, lo que generó, grosso modo, un ajuste con la nota alcanzada. Sin embargo, lo más relevante de la propuesta fue el interpelar la concepción tradicional de la evaluación y la consideración de la autoevaluación como un nuevo proceso de aprender a evaluar, pues consideramos que es autoevaluándonos como aprendemos sobre evaluación. Esto es, hacerlo sobre sí mismo permite la trasferencia a otras situaciones.

Consideramos que las instancias ofrecidas en este dispositivo promueven una evaluación continua: la autoevaluación, la coevaluación, la del docente - escrita u oral- involucran momentos de reflexión de los estudiantes sobre sus propios modos de enseñar y de aprender, así como de lo aprendido. Asimismo, respecto a la evaluación, actualmente nos encontramos repensando el complejo tema de la calificación grupal en relación a la distribución de los roles desempeñados por los estudiantes en la realización de la clase. Una de las cuestiones que encontramos, y que queda por repensar también, son las evaluaciones entre pares. Una de las formas para llevarlas podría consistir en que las retroalimentaciones entre compañeros fueran anónimas para fomentar, de ese modo, que puedan expresar sus comentarios sin verse inhibidos por el hecho de «criticarse» mutuamente. La implementación de este dispositivo se adapta a los tiempos institucionales, pero creemos necesario explicitar que la posibilidad de una segunda vuelta en «dar clase» implicaría una mayor articulación entre los conocimientos adquiridos y la aplicación de los mismos, dando cuenta de una auténtica internalización, favoreciendo la autonomía en el aprendizaje y el cambio en la concepción de la evaluación.

Para finalizar, concluimos que, a pesar de las falencias mencionadas, el dispositivo brinda a los estudiantes la posibilidad de «reflexionar sobre el tipo de docentes que quieren ser, sobre el tipo de aula que quieren construir para sus futuros alumnos y sobre el tipo de proyecto educativo que intentan proponer» (De Laurentis, 2013, p. 245). 


\section{Referencias}

Álvarez Méndez, J. (2001a). Evaluar para conocer, examinar para excluir. Madrid: Editorial Morata.

Álvarez Méndez, J. (2001b). Evaluación a examen. Madrid: Miño y Dávila Editores.

Álvarez, Z., y Porta, L. (2012). Caminos de indagación sobre la buena enseñanza: aproximación biográfica-narrativa en educación superior. Revista de Educación, III(4), 75-88.

Álvarez, Z., Portal, L., y Sarasa, M. (2010). La investigación narrativa en la eenseñanza: buenas prácticas y las biografías de los profesores memorables. Revista de Educación, I(1), 159-179.

Anjjovich, R. (comp.). (2010). La evaluación significativa. Buenos Aires: Paidós.

Bruner, J. (1991). Actos de significado. Más allá de la revolución cognitiva. Madrid: Alianza.

Burbules, N. (1999). El diálogo en la enseñanza: teoría y práctica. Buenos Aires: Amorrortú.

Camilloni, A. (2001). Sistemas de calificación y regímenes de promoción. En A. Camilloni, S. L. Celman, y M. D. Paloú, La evaluación de los aprendizajes en el debate didáctico contemporáneo (pp. 133-176). Buenos Aires: Paidós.

Canale, M. (2013). Cuadernillo "Cómo enseñamos». Material de la Cátedra Adolescencia, Educación y Cultura. Mar del Plata: UNMDP.

Carlino, P. (2005). Escribir, leer y aprender en la Universidad. Una introducción a la alfabetización académica. Buenos Aires: F.C.E.

Celman, S. (2006). Evaluando los sentidos de la evaluación. Confrencia dictada en curso de posgrado: "La evaluación en la universidad». UNER-UNL.

Celman, S. (2001). ¿Es posible mejorar la evaluación y transformarla en herramienta de conocimiento? En A. Camilloni, S. L. Celman, y M. D. Paloú, La evaluación de los aprendizajes en el debate didáctico contemporáneo. Buenos Aires: Paidós.

Celman, S., Galarraga, G., Gerard, A., Grinóvero, N., Martínez, M., Olmedo, V., y Rafaghelli, M. (2015). Experiencias entre la universidad pública y los Institutos de Formación Docente. Entre Ríos: Editorial Eduner.

Clandinin, D. (2006). Narrative Inquiri: A Methodology for Studying Lived Experience. Studies in Music Education(27), 44-54.

Clandinin, D., Steeves, P., y Chung, S. (2008). Creando espacios de investigación narrativa en la formación del profesorado. En L. Porta, y M. Sarasa, Formación y desarrollo de la formación docente en el profesorado: las buenas prácticas y sus narrativas (pp. 59-83). Mar del Plata: uNMDP.

De Laurentis, C. (2013). Estudiantes narrando sus clases: relatos en el profesorado de inglés de la unMDP. Revista de Educación, IV(6), 231-248.

Díaz Barriga, A. (2001). El examen: textos para su historia y debate. En A. Camilloni, S. L. Celman, y M. D. Paloú, La evaluación de los aprendizajes en el debate didáctico contemporáneo. Buenos Aires: Paidós. 
Dussel, I. (2011). Aprender y enseñar en la cultura digital. Buenos Aires: Fundación Santillana.

Erickson, F. (1997). Métodos cualitativos de investigación sobre la enseñanza. En M. Wittrock, La investigación de la enseñanza II. Métodos cualitativos y de observación. Barcelona: Paidós.

González, P. (2000). La evaluación del aprendizaje de los estudiantes en la enseñanza universitaria. La Habana: CEPES.

Goodson, I. (2005). Hacia un desarrollo de las historias personales y profesionales de los docentes. Revista Mexicana de Investigación Educativa, (19), 733-758.

Gort, A. (2008). Diagnóstico y transformaciones en la evaluación del aprendizaje: un estudio en la Facultad de Biología de la Universidad de La Habana [Tesis de doctorado]. La Habana: CEPES.

Litwin, E. (2001). La evaluación: campo de controversias y paradojas o un nuevo lugar para la buena enseñanza. Buenos Aires: Paidós.

Mc Ewan, H. (2005). Las narrativas en el estudio de la docencia. En H. Mc Ewan, y K. Egan, La narrativa en la enseñanza, el aprendizaje y la investigación. Buenos Aires: Amorrortu.

Sampaio, E. (2012). La evaluación mediadora formativa. Una experiencia en el contexto de la Educación Superior en Brasil. Revista de Ciencias Pedagógicas, (1), 1-11.

Senado y Cámara de Diputados de la Nación de Argentina. (2010). Ley 26.618 de 2010: Matrimonio Civil. Código civil, modificación. Diario oficial.

Senado y Cámara de Diputados de la Nación de Argentina. (2010). Decreto 1054 de 2010: por el cual se promulga la Ley 26.618 de 2010. Diario oficial.

Vain, D. (2012). El enfoque interpretativo en investigación educativa: algunas consideraciones teórico-metodológicas. Revista de Educación, III(4), 37-45.

Valles, M. (2000). Técnicas cualitativas de investigación social. Reflexión metodológica y práctica profesional. Madrid: Editorial Síntesis.

Watzlawick, P., Beavin, J., y Jackson, D. (1985). Teoría de la comunicación humana. Barcelona: Editorial Herder.

Weissman, P. (2013). Cuadernillo «Cómo aprendemos». Material de la Cátedra "Adolescencia, Educación y Cultura». Mar del Plata: Universidad Nacional de Mar del Plata.

Weissman, P. (2013). Cuadernillo "Teorías de aprendizaje». Material de la Cátedra "Adolescencia, Educación y Cultura». Mar del Plata: Universidad Nacional de Mar de Plata.

Weissman, P. (2013). Nuevas tendencias en evaluación. Relato de una experiencia. I Encuentro Internacional de Educación (pp. 1-9). Tandil: Universidad Nacional de Mar del Plata.

Weissman, P. (2013). Propuesta de trabajo docente de la asignatura «Adolescencia, Educación y Cultura» (Inédito). Mar del Plata. 
Recibido: 21 de septiembre 2016

Aceptado: 19 de noviembre 2016

Cómo citar: Alquézar, M., y Buzeki, M. (2017) Saber y hacer: evaluación de la transferencia de conocimientos didácticos a la práctica docente. Praxis Pedagógica, 20, 63-84 